\title{
Respiratory function in the prune-belly syndrome
}

\author{
Charles H Crompton, Ian B MacLusky, Denis F Geary
}

\begin{abstract}
Respiratory function was evaluated in 11 patients with prune-belly syndrome. Nine had evidence of gas trapping and six of restrictive lung disease. These abnormalities of lung function appear to be secondary to the musculoskeletal disorder associated with prune-belly syndrome rather than parenchymal lung disease.
\end{abstract}

(Arch Dis Child 1993; 68: 505-506)

Prune-belly syndrome is a congenital disorder that classically consists of deficient abdominal musculature, urinary tract abnormality often associated with renal hypoplasia or dysplasia, and cryptorchidism. Pulmonary hypoplasia secondary to oligohydramnios can result in varying degrees of respiratory insufficiency in the neonatal period, and clinical experience suggests that during childhood respiratory illness is common. Results of respiratory function testing have not been reported nor the pulmonary abnormality characterised in these patients. We therefore measured pulmonary function in a group of children and adolescents with prunebelly syndrome to determine the frequency and severity of respiratory dysfunction.

\section{Subjects and methods}

We studied 11 males aged 7-19 years with prunebelly syndrome; three had renal allografts. Details of respiratory symptoms obtained by history from patient or parents were recorded. Chest $x$ ray films were available for 10 of the patients. Renal function assessment was by serum creatinine with estimation of glomerular filtration rate by the equation of Schwartz et al. ${ }^{\prime}$

Respiratory function assessments were performed in the pulmonary function laboratory of the Division of Respiratory Medicine. Spirometry measurements included forced vital capacity (FVC), forced expiratory volume in one second $\left(\mathrm{FEV}_{1}\right)$, and maximum mid-expiratory flow (MMEF). Performance of these testing procedures was based on the American Thoracic Society recommendations. ${ }^{2}$ The results were selected from the highest sum of FVC plus FEV from acceptable curves. Lung volumes (total lung capacity, TLC and residual volume, RV) were measured both by helium dilution and constant volume body plethysmography (PK Morgan Ltd). Maximum inspiratory pressures (MIPS) and maximum expiratory pressures (MEPS) were determined according to previously described techniques. ${ }^{3}$

Spirometry measurements were recorded as a percentage of the expected value for the patient's height and sex. ${ }^{+}$Arm span instead of height was used to calculate expected values for the one patient with significant scoliosis. MIPS and
MEPS measurements were compared with previously published normative data.

\section{Results}

Three patients had symptoms consistent with lower airway disease. The remaining patients gave no history of respiratory disease.

Radiographs were normal in patients 2 and 8. Pectus carinatum or excavatum was present in all other patients. Lung volumes were increased in patients 3 and 10 . Thoracic scoliosis was present in patients $5,6,7$, and 11 and was mild in those except patient 11 who had a severe deformity.

Details of the subjects are shown in the table. Patient 3 demonstrated normal lung function. All except two showed evidence of gas trapping with raised $(>30 \%)$ ratio $R V$ : TLC. Of these, only patient 11 had evidence of peripheral airway obstruction on spirometry with $\mathrm{FEV}_{1} 40 \%$ and MMEF $29 \%$ of predicted. The remaining eight patients did not have evidence for primary peripheral airway disease on spirometry, although patient 8 had findings suggestive of a mild mixed restrictive/obstructive pattern. Six of the 11 patients also had varying degrees of restrictive lung disease $(2,5,6,7,9$, and 11$)$. In four of the 11 patients with prune-belly syndrome MIPS fell below the expected range and in three MEPS were low. Of those within the normal range, all had values at the low end of the normal range.

Patient 6 had lung function studies before and after transplant. With a well functioning graft six months after transplant, there was no significant change in the restrictive abnormality (pretransplant values not shown in table).

\section{Discussion}

There are many reasons why respiratory function might be abnormal in prune-belly syndrome, including pulmonary hypoplasia secondary to in utero oligohydramnios and disordered thoracic mechanics due to scoliosis, rib cage abnormalities, and abdominal muscle weakness. For the $30 \%$ or so patients with prune-belly syndrome who develop chronic renal failure, pulmonary function may be further impaired as a consequence of uraemia. Respiratory function may be further compromised when mechanics are altered such as during peritoneal dialysis, anaesthesia, and surgery. ${ }^{5}$

We have documented abnormal lung function in the majority of patients with prune-belly syndrome studied, irrespective of the presence of renal impairment. Most of the patients had a raised RV:TLC ratio, indicating gas trapping. Flow-volume loops indicated that this was likely due to peripheral airway disease in only one patient (number 11), who had previously been diagnosed as having asthma. In the remaining 
Clinical characteristics and results of respiratory function testing in the patients with prune-belly syndrome

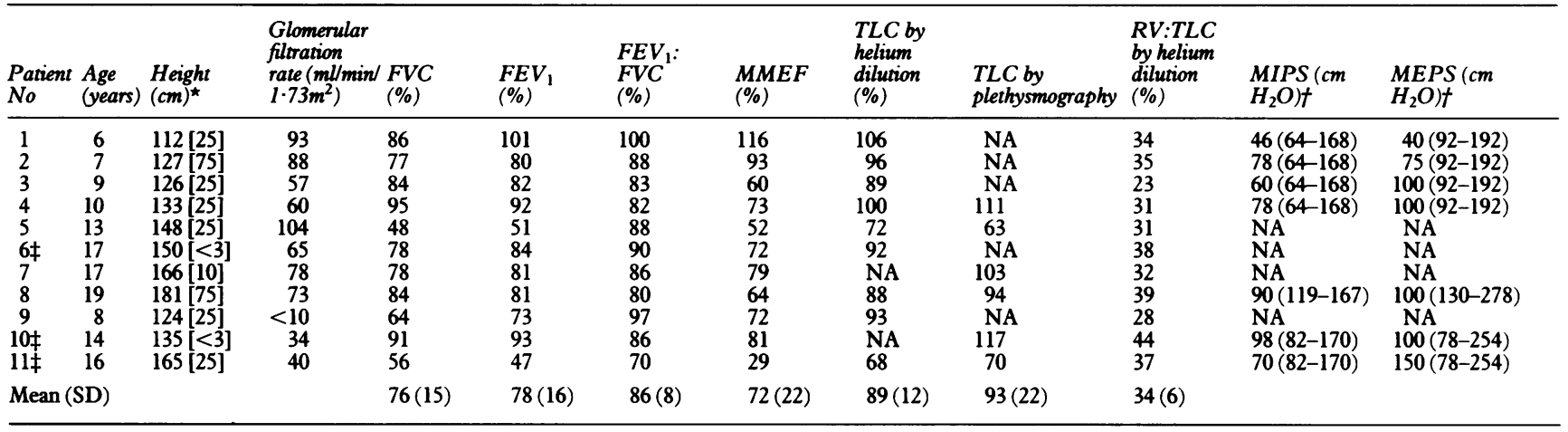

*Centile ranking in square brackets.

tReference range in parentheses.

†Renal allograft recipients.

NA $=$ not available

FVC, FEV 1 , MMEF, and TLC expressed as percent of predicted.

patients, flow-volume loops failed to demonstrate any evidence of peripheral airway obstruction. The gas trapping in the other patients appeared to be secondary to poor expiratory effort as a consequence of the deficient abdominal musculature. Poor muscle effort in many of the patients with prune-belly syndrome is further suggested by the finding of low MIPS and MEPS, tests which reflect muscular effort in generating inspiratory and expiratory pressures. The restrictive defect in the patients with prunebelly syndrome thus appears to be secondary largely to the musculoskeletal abnormality rather than due to an interstitial lung problem. However, the patients that develop chronic renal failure could also be expected to develop lung function abnormalities that are associated with uraemia. ${ }^{6}$

This study confirms and further defines a previously reported abnormality of respiratory function in patients with prune-belly syndrome.
It appears that most, if not all, individuals with prune-belly syndrome can be expected to have abnormal lung function, and half may show significant restrictive lung disease. The detection of abnormal respiratory function in asymptomatic patients emphasises the need for caution when patients with prune-belly syndrome are subjected to anaesthesia or surgery, particularly if there is coincident renal impairment.

1 Schwartz GJ, Brion LP, Spitzer A. The use of plasma creatinine concentration for estimating glomerular filtration rate in infants, children, and adolescents. Pediatr Clin North Am 1987; 34: 571-90.

2 American Thoracic Society. Standardisation of spirometry 1987 update. Am Rev Respir Dis 1987; 136: 1285-98.

3 Szeinberg A, Marcotte JE, Roizin H, et al. Normal values of maximal inspiratory and expiratory pressures with a portable apparatus in children, adolescents, and young adults. Pediatr Pulmonol 1987; 3: 255-8.

4 Weng T-R, Levison $H$. Standards of pulmonary function in children. Am Rev Respir Dis 1969; 99: 879-94.

5 Katz J, Steward DJ, eds. Anesthesia and uncommon pediatric diseases. Philadelphia: WB Saunders, 1987: 200-2.

6 Prezant JP. Effect of uremia and its treatment on pulmonary function. Lung 1990; 168: 1-14. 